\title{
A note on zero-sets in the Stone-Čech compactification
}

\section{Rudd}

\begin{abstract}
The ring $C(X)$ is the ring of all continuous real-valued functions on a completely regular Hausdorff space $X$, and $\beta X$ is the Stone-Čech compactification of $X$.

The author proves a theorem which leads to a characterization of those zero-sets in $X$ whose closures (in $B X$ ) are zero-sets in $B X$, and relates this characterization to the ideals in the ring $C(X)$.
\end{abstract}

\section{Introduction}

If $X$ is a space in which $C(X)$ only has bounded members, that is, $X$ is pseudocompact, then

(1) the uniform closure of any ideal in $C(X)$ is the same as its $m$-closure, and

(2) for any function $f \in C(X)$,

$$
\mathrm{cl}_{\beta X}\{x \in X \mid f(x)=0\}=\left\{p \in \beta X \mid f^{*}(p)=0\right\},
$$

where $f^{*}$ denotes the extension of $f$ to BX. (See [1, $7 Q]$.

Indeed, both (1) and (2) are each equivalent to pseudocompactness of $X$. In this note we consider the case in which $X$ is not pseudocompact. We then characterize those functions for which (2) does not hold and show that these are precisely the functions which "cause" (1) not to hold. We

Received 18 November 1974. 
also obtain necessary and sufficient conditions for the closure (in $B X$ ) of a zero-set in $X$ to be a zero-set in $B X$. (See $[1,6 \mathrm{E} .2]$.

\section{Preliminaries}

The reader is referred to [1] and [2, 52] for background information and notations.

We shall let $X$ denote an arbitrary completely regular Hausdorff space. (of course, if $X$ is pseudocompact, the assertions in this paper are vacuous.)

We point out that for $f \in C(X), Z(f)$ denotes $\{x \in X \mid f(x)=0\}$, and $Z\left(f^{*}\right)$ denotes $\left\{p \in \beta X \mid f^{*}(p)=0\right\}$. If $f \in C^{*}(X)$, that is, if $f$ is bounded, then $f^{*}$ is denoted by $\hat{f}$.

For a subset $S$ of $\beta X$, we denote ${ }^{c} I_{\beta X}(S)$ by $S^{\beta}$.

For an ideal $I$ of $C(X), I^{U}$ denotes the uniform closure of $I$, and $I^{m}$ denotes the $m$-closure. (See $[2,2.4]$.)

DEFINITION 1.1. Let $f \in C(X)$. Then a subset $A$ of $X$ is said to be a near-zero set for $f$, if for any $\delta>0$, there is an $a \in A$ with $|f(a)|<\delta$.

THEOREM 1.2. Let $f \in C(X)$. Then the following are equivalent:

(i) $Z\left(f^{*}\right) \neq Z(f)^{\beta}$;

(ii) there is a near-zero set for $f$ which is completely separated from $Z(f)$;

(iii) there is a maximal ideal $M$ in $C(X)$ so that $f \in M^{\mu} \backslash M$;

(iv) there is an ideal $I$ in $C(X)$ so that $f \in I^{\mu} \backslash I^{m}$.

Proof. $(i) \rightarrow(i i)$. Since $Z\left(f^{*}\right) \supseteq Z(f)^{\beta}([1,7.11])$, the hypothesis implies that there is a $p \in Z\left(f^{*}\right) \backslash Z(f)^{\beta}$. Hence there is a neighborhood $W$ of $p$ so that $W^{\beta} \cap Z(f)^{\beta}=\varnothing$. Let $A=W \cap X$, and consider $\delta>0$. Since $\left\{p \in B X \mid-\delta<f^{*}(p)<\delta\right\} \cap W$ is an open (in $B X$ ) neighborhood of $p$, it follows that $\left(f^{*}\right)^{-1}(-\delta, \delta) \cap(W \cap X) \neq \emptyset$, and hence $A$ is a near- 
zero set for $f$. Also, since $W^{\beta}$ and $Z(f)^{\beta}$ are completely separated in $B X, A$ and $Z(f)$ are completely separated in $X$.

(ii) $\rightarrow$ (iii). Let $A$ be a near-zero set for $f$ which is completely separated from $Z(f)$. Then the closures $A^{\beta}$ and $\dot{Z}(f)^{\beta}$ are disjoint in $B X$. (See $[1,6.5$ III].) For each $\delta>0$, let

$$
F_{\delta}=A^{\beta} \cap\left\{p \in B X \mid-\delta \leq f^{*}(p) \leq \delta\right\} .
$$

By the compactness of $B X$, there is a $p \in \cap\left\{F_{\delta} \mid \delta>0\right\}$, and this $p$ has the property that $f^{*}(p)=0$. Since $p \in A^{\beta}, p \notin z(f)^{\beta}$, and we have, using $[2,2.4]$, that $f \in\left(M^{p}\right)^{u} M^{p}$.

$(i i i) \rightarrow(i)$. If $f \in\left(M^{p}\right)^{\mathcal{U}} \backslash^{p}$, then $p \in Z\left(f^{*}\right) \backslash Z(f)^{\beta}$. The equivalence of (iii) and $(i v)$ follows from $[2,5.2]$ and $[1,7 Q .2]$.

COROLLARY 1.3. Let $f \in C(X)$. Then $z(f)^{\beta}$ is a zero-set in $B X$ if and only if there is a $g \in C^{*}(X)$ so that $Z(f)=Z(g)$, and no nearzero set for $g$ is completely separated from $Z(g)$. In this case, $Z(f)^{\beta}=Z(\hat{g})$. Furthermore, given the zero-set $Z(\hat{g})$ in $\beta X$, it is of the form $Z(f)^{\beta}$ for some $f \in C(X)$ if and only if no near-zero set for $g$ is completely separated from $Z(g)$.

Proof. Suppose $Z(f)^{\beta}$ is a zero-set in $\beta X$, say $Z(f)^{\beta}=Z(\hat{g})$. Then, intersecting with $X$, we have that $Z(f)=Z(g)$, from which it follows that $z(g)^{\beta}=Z(\hat{g})$. By Theorem 1.2 , no near-zero set for $g$ can be completely separated from $Z(g)$.

Conversely, if $g \in C^{*}(X)$ which satisfies the hypotheses of the corollary, then it follows by Theorem 1.2 that $z(g)^{\beta}=z(\hat{g})$, and hence $z(\hat{g})=z(f)^{\beta}$.

The rest of the corollary follows easily.

EXAMPLE 1.4. Let $X$ denote the non-negative reals and define $f(x)=x$ on $0 \leq x \leq 1$ and $f(x)=1 / x$ for $x \geq 1$. Then $[1, \infty)$ is a near-zero set for $f$ which is completely separated from $Z(f)$, and 
hence $Z(f)^{\beta} \neq Z(\hat{f})$. However $Z(f)^{\beta}=\{0\}$, a zero-set in $B X$. Thus $z(f)^{\beta}$ can be a zero-set in $\beta X$, even if it is not the zero-set of $f^{*}$.

EXAMPLE 1.5. Consider the sine function on the non-negative reals. Since $Z$ (sine) is countable and discrete, it follows that there is a nearzero set for sine which is completely separated from $Z$ (sine), and hence sine $\epsilon M^{\mu} \backslash$ for some maximal ideal $M$. Also $2(\text { sine })^{\beta}$ can not be a zero-set in $B X$, because if $Z($ sine $)=Z(g)$ for any $g \in C^{*}(X)$, then there would be a near-zero set for $g$ which is completely separated from $z(g)$.

REMARK 1.6. It is believed that the equivalence of condition (2) in the introduction with pseudocompactness of $X$ is well known, but the author could find no direct reference. A proof could easizy be written based on Theorem 1.2 .

\section{References}

[1] Leonard Gillman and Meyer Jerison, Rings of continuous functions (Van Nostrand, Princeton, New Jersey; Toronto; London; New York; 1960).

[2] David Rudd, "On isomorphisms between ideals in rings of continuous functions", Trans. Amer. Math. Soc. 159 (1971), 335-353.

\footnotetext{
Department of Mathematics,

old Dominion University,

Norfolk,

virginia,

USA.
} 